\title{
Impulse electromagnetic drive for magnetic energy accumulation press
}

\author{
V.Yu. Neyman \\ Novosibirsk State Technical University \\ Novosibirsk, Russian Federation \\ e-mail:nv.nstu@ngs.ru
}

\author{
A. V. Markov \\ Novosibirsk State Technical University \\ Novosibirsk, Russian Federation \\ e-mail: markov2017@ngs.ru
}

\begin{abstract}
Perspectives of preliminary magnetic energy accumulation in a linear electromagnetic drive inductances system have been considered. This magnetic energy accumulation mode provides the increase of final speed, striker impact energy and electric drive efficiency. The electromagnetic drive circuit variant where magnetic energy is accumulated when the striker is restrained at initial acceleration interval has been analyzed.

The basic demands for the magnetic energy accumulation mode have been formulated. The effective methods of magnetic energy accumulation have been developed. The methods allow to increase twice and more the impact energy and efficiency of impulse electromagnetic drives.
\end{abstract}

Keywords - impulse electromagnetic drive; electromagnetic motor; magnetic energy accumulation; efficiency; impact energy.

\section{INTRODUCTION}

Mining, construction work, geological exploration, immersion in the anchor ground, piles and other special impulse technologies are connected with the use of impact machines [1-4].

The use of electromagnetic pulse machines for these purposes simplifies the process of shock action on deformable objects and environment [5-9].

The machines and mechanisms based on impulse electromagnetic drives have relatively high reliability, increased service life, low cost in production and exploitation.

Methods for electromagnetic drives design are well known but continue to be improved [10-16].

Creation and improvement of impact machines with high impact energy are perspective due to new control methods. One of these control methods is the momentary retention of the striker when the voltage is applied to the winding of the electromagnetic motor.

When the striker is held, the starting time and the current increase, and the movement time decreases. The analysis of the linear electromagnetic motor (LEMM) spring return process shows that it is possible to increase in some degree the dynamic efficiency of the motor by increasing its speed of movement and mechanical energy at the output by increasing the counteracting force.
One of the effective methods for boosting electromagnetic motors power is the accumulation of magnetic energy in the system of inductances during the operating cycle.

Practical realization of the accumulation of magnetic energy in the inductances of the motor during the operating cycle consists in the artificial retention of the striker with a counter-acting force. An experimental test on physical models (Fig. 1) showed that an increase of the counteracting force at the start-up stage in linear electromagnetic motors makes it possible to improve their specific power and energy characteristics.

The implementation of such device can be simpler as it is shown in Fig. 1.

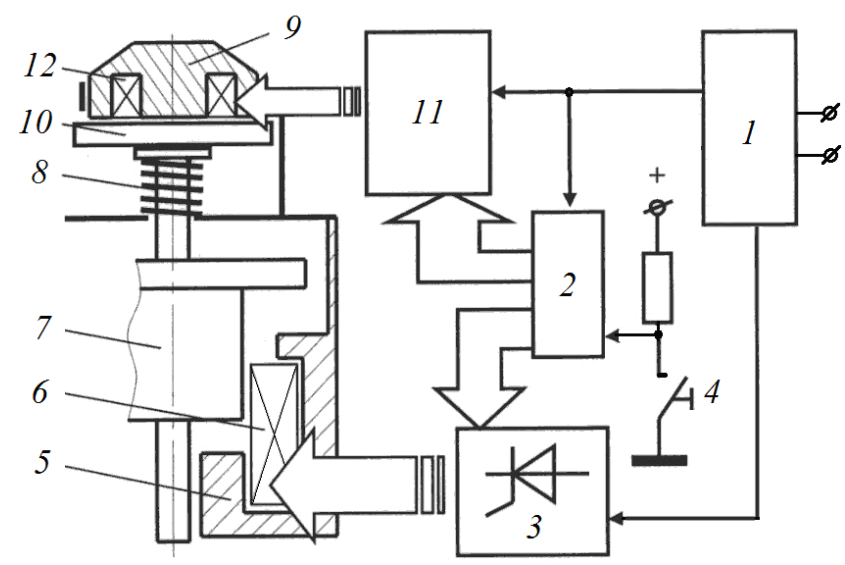

Fig. 1. Electromagnetic actuator with retaining striker

The device (Fig. 1) includes the following parts: DC power supply 1 , control pulse generating unit 2 , thyristor converter of working coil 3, control panel 4 and LEMM magnetic circuit 5, excitation winding 6 , striker 7 , return spring 8 . An arm restraint device is made on the basis of the electromagnet 9 with the external pulling armature 10.

The electromagnet is controlled by means of the power supply and control device 11 by applying a voltage pulse of specific width to the excitation winding 12 . 
The magnetic energy accumulation methods were implemented in different impact machine electric drive construction variants (Fig. 2-5).

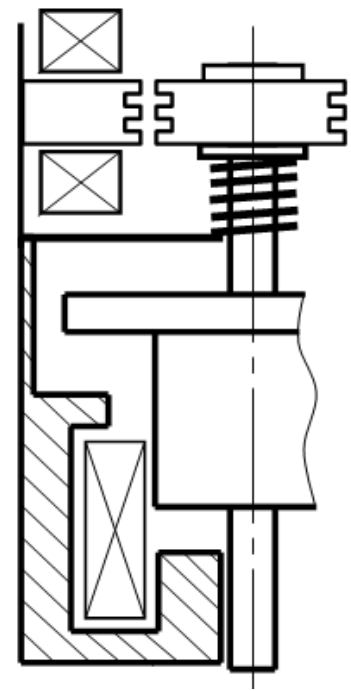

Fig. 2. Impact electric drive implementation, variant 1

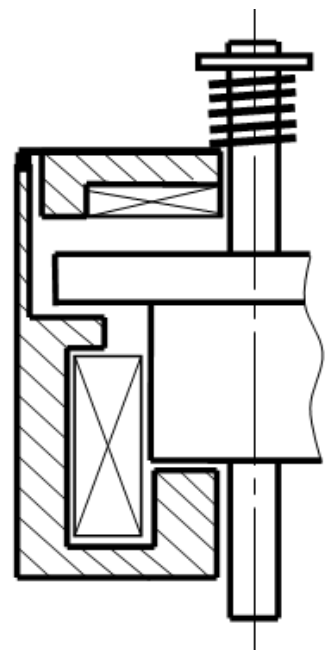

Fig. 3. Impact electric drive implementation, variant 2

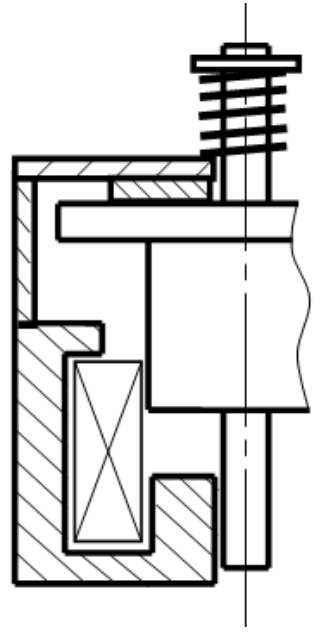

Fig. 4. Impact electric drive implementation, variant 3

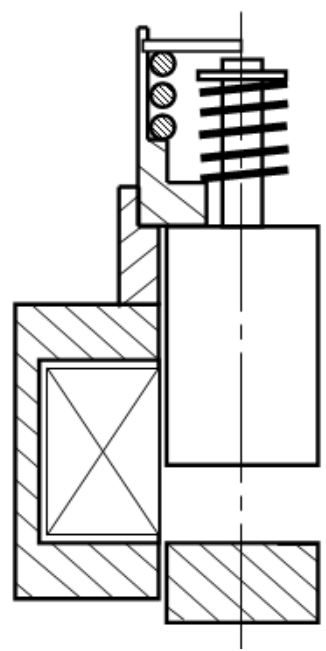

Fig. 5. Impact electric drive implementation, variant 4

\section{METHODS OF ANALYSIS}

To determine the influence of the retaining force of the striker on the energy and time characteristics of a LEMM, the equations of the balance of forces acting on the striker are considered. When the mobile system is at rest, the equation of the balance of forces of the mechanical system is described by equations:

$$
\begin{gathered}
F_{\mathrm{em}}(x)-\left.F_{\mathrm{cf}}(x)\right|_{t_{\mathrm{fr}}=t_{1}} ^{=0}, \\
\left.m \frac{d^{2} x}{d t^{2}}\right|_{t_{\mathrm{fr}}=t_{1}}=0
\end{gathered}
$$


where $m$ is the striker mass, $x$ is the current coordinate of displacement, $F_{\text {em }}(x), \quad F_{\text {cf }}(x)$ are electromagnetic and opposing forces.

After the beginning of the movement, the equation of the balance of forces is described by the equation of motion of the mechanical system:

$$
m \frac{d^{2} x}{d t^{2}}=F_{\mathrm{em}}(x)-F_{\mathrm{cf}}(x)
$$

The efficiency of converting electromagnetic energy into mechanical work during the movement of the striker is determined by the characteristics of the flux linkage and the working gap.

For a linear magnetic system, the mechanical work of the motor is represented for an elementary magnetic cycle:

$$
A=\frac{1}{2}\left(i_{1} \varpi_{2}-i_{2} \varpi_{1}\right),
$$

where $i_{1}, \amalg_{1}$ are the initial value of current and flux linkages corresponding to the moment of starting, $i_{2}, \omega_{2}$ are the values of these same quantities at the end of the movement.

During the motion, the electromagnetic motor overcomes the opposing force $F_{\mathrm{cf}}(x)$. The current at which the pulling force $F_{\mathrm{em}}(x)$ becomes equal to the opposing $F_{\mathrm{cf}}(x)$, determines the beginning of the movement of the striker and the starting current. When the current is lower than the starting current, the striker is at rest.

The current and flux linkage at the moment of starting are:

$$
\begin{gathered}
i_{1}=\sqrt{2 F_{0} x_{1} / L_{1}}, \\
ш_{1}=\sqrt{2 F_{0} x_{1} L_{1}},
\end{gathered}
$$

where $L_{1}$ is the initial inductance corresponding to the initial operating air gap $x_{1}, F_{0}$ is the resistance force to movement of the striker at the time of starting.

Assumed that the reaction force is changed as follows:

$$
F_{\Pi}=\left\{\begin{array}{c}
F_{0} \text { at } t \leq t_{0} \text { and } x=x_{1} \\
F_{\mathrm{cf}}(x) \text { at } t \geq t_{0} \text { and } x \leq x_{1} .
\end{array}\right.
$$

The equation of the energy balance of a mechanical system during the movement of the striker has the form:

$$
\int_{v_{1}}^{v_{2}} m v d v=\int_{x_{1}}^{x_{2}} F_{\mathrm{em}}(x) d x-\int_{x_{1}}^{x_{2}} F_{\mathrm{cf}}(x) d x
$$

where $v_{1}, v_{2}$ are the initial and final speed of the striker.

The first term on the right-hand side of (6) determines useful mechanical work on the displacement interval $x_{1} \ldots x_{2}$ :

$$
\int_{x_{1}}^{x_{2}} F_{\mathrm{em}}(x) d x=\frac{1}{2}\left(i_{1} \varpi_{2}-i_{2} \amalg_{1}\right) .
$$

The electromagnetic force $F_{\mathrm{em}}(x)$ static force is equal to $F_{\text {cf }}(x)$. The kinetic energy of the striker at the end of the stroke is $m \frac{d^{2} x}{d t^{2}}=0$.

Then (6) takes the form:

$$
\int_{x_{1}}^{x_{2}} F_{\mathrm{em}}(x)=\int_{x_{1}}^{x_{2}} F_{\mathrm{cf}}(x) d x
$$

In this case, the entire energy of the magnetic field is converted into mechanical work. As it follows from (8), the greater the force $F_{\mathrm{cf}}(x)$, the more is the strength $F_{\mathrm{em}}(x)$.

If (8) is not satisfied, then at the end of the stroke the striker achieves certain speed $v_{2}$. In this case, the driving force is equal to dynamic thrust force. The (6) after integrating the left-hand side can be represented in the form:

$$
\frac{m v_{2}^{2}}{2}-\frac{m v_{1}^{2}}{2}=\int_{x_{1}}^{x_{2}} F_{\mathrm{em}}(x) d x-\int_{x_{1}}^{x_{2}} F_{\mathrm{cf}}(x) d x
$$

According to the initial condition $v_{1}=0$, then:

$$
\frac{m v_{2}^{2}}{2}=\int_{x_{1}}^{x_{2}} F_{\mathrm{em}}(x) d x-\int_{x_{1}}^{x_{2}} F_{\mathrm{cf}}(x) d x
$$

Replacing the first term on the right-hand side of (9) by (7) and transformations with respect to (3) and (4) gives:

$$
\frac{m v_{2}^{2}}{2}=\frac{1}{2} \sqrt{2 F_{0} x_{1}}\left(\omega_{2} \sqrt{\frac{1}{L_{1}}}-i_{2} \sqrt{L_{1}}\right)-\int_{x_{1}}^{x_{2}} F_{\mathrm{cf}}(x) d x
$$

When $i=$ const $, i_{1}=i_{2}, \quad \omega_{2}=L_{2} i_{2}=L_{2} i_{1}$ (10) takes the form:

$$
\frac{m v_{2}^{2}}{2}=F_{0} x_{1}\left(k_{\mathrm{L}}-1\right)-\int_{x_{1}}^{x_{2}} F_{\mathrm{cf}}(x) d x
$$


where $k_{\mathrm{L}}=\frac{L_{2}}{L_{1}}$.

The first term on the right-hand side of (11) determines the work of the electromagnetic forces performed when the striker moves from the initial position to the final position.

This work is spent on overcoming the strength of resistance to movement and the accumulation of kinetic energy. If the striker is moved from the $x_{1}$ to $x_{2}$, then $F_{c f}(x)=0$ and the total work of electromagnetic forces is expended to the movement of the striker:

$$
\int_{x_{1}}^{x_{2}} F_{\mathrm{em}}(x) d x=\frac{m v_{2}^{2}}{2}=F_{0} x_{1}\left(k_{\mathrm{L}}-1\right) .
$$

From (7), (11) and (12) it is evident that the greater resistance to movement of the striker $F_{0}$ at the moment of starting takes place, the greater energy is concentrated in the volume of the electromagnetic machine.

The increase of energy at the end of the energy conversion process is associated with the increase of the starting current and the energy accumulated in the magnetic field when the striker begins to move. From (12) it is possible to determine the final speed of the striker:

$$
v_{2}=\sqrt{\frac{2 F_{0} x_{1}\left(k_{\mathrm{L}}-1\right)}{m}} .
$$

Assuming that the movement of the striker is uniformly accelerated, its travel time is defined as:

$$
\begin{gathered}
t_{\mathrm{m}}=\frac{x_{1}-x_{2}}{v_{2}}=\frac{x_{1}}{\sqrt{\frac{2 F_{0} x_{1}\left(k_{\mathrm{L}}-1\right)}{m}}}= \\
=\sqrt{\frac{x_{1} m}{2 F_{0}\left(k_{\mathrm{L}}-1\right)}} .
\end{gathered}
$$

It follows from (14) that when the holding force is increased at the moment when the striker starts, the time of its movement decreases, which ensures the high final speed $v_{2}$ and kinetic energy.

Fig. 6 shows the examples of electromagnetic drive specific impact energy dependences on striker retention force.

Fig. 7 presents the electric drive efficiency dependence on striker retention force for the variant in Fig. 1.

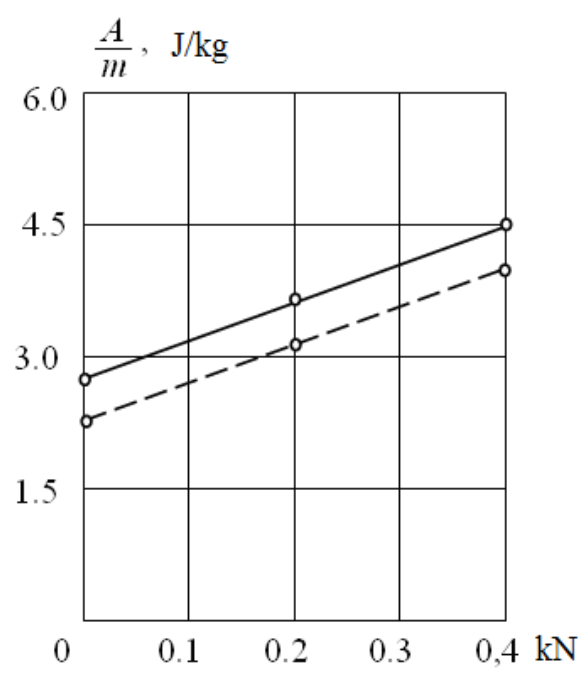

Fig. 6. Specific impact energy dependences on striker retention force

Efficiency, \%

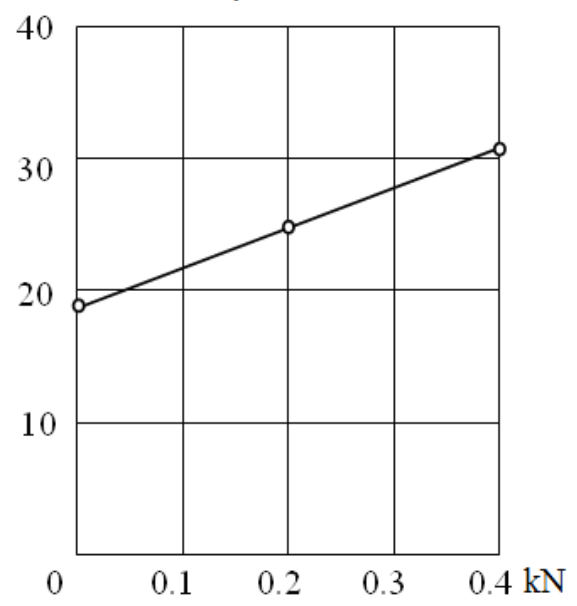

Fig. 7. Electric drive efficiency dependence on striker retention force

\section{CONCLUSION}

Analysis of (13) and (14) allows formulating the basic requirements for electromagnetic machines with the held striker. The striker holding devices must provide significant resistance to movement at the beginning of the stroke and minimum resistance to movement on the rest of the stroke. The possibility of adjusting the resistance force to the movement at the beginning of the stroke makes it possible to consume small energy from a source.

Effective ways of accumulating magnetic energy in electromagnetic shock machines of various designs have been developed to significantly increase power and energy performance.

The impact energy for experimental electromagnetic machines increased from 2 to 4 times and achieved $9 \ldots 10$ $\mathrm{J} / \mathrm{kg}$. The efficiency of electromagnetic machines has doubled and approached $50 \%$. 


\section{References}

[1] B.F. Simonov, S.V. Serdyukov, E.N. Cherednikov, "Results of research and field work on the rose-oil sheniyu vibroseis method", Oil Industry, Num. 5, p. 48, 1996.

[2] V.N. Oparin, B.F. Simonov, "Nonlinear deformation-wave processes in the vibrational oil geotechnologies", Journal of Mining Science, vol. 46, Num. 2, pp. 95-112, 2010.

[3] M.V. Kurlenya, S.V. Serdyukov, "Intensification of oil production at the low-frequency impact vibroseismic" Mining informational and analytical bulletin, Num 5, pp. 29-34, 2004.

[4] K.M. Usanov, A.V. Volgin, E.A. Chetverikov, V.A. Kargin, A.P Moiseev, Z.I. Ivanova, "Power electromagnetic strike machine for engineering-geological surveys", IOP Conference Series: Earth and Environmental Science [Electronic resource], p. 032049, 2017.

[5] K.M. Usanov, A.V. Volgin, E.A. Chetverikov, V.A. Kargin, A.P. Moiseev, Z.I. Ivanova, "Strike action electromagnetic machine for immersion of rod elements into ground", IOP Conference Series: Earth and Environmental Science [Electronic resource], p. 032050, 2017.

[6] K.M. Usanov, G.G. Ugarov, V.I. Moshkin, Linear impulse electromagnetic drive of machines with self-contained power supplies, Kurgan: Kurgan University, p. 284, 2006.

[7] V.P. Pevchev, "The superexitation and efficiency relation in a shortstroke pulsed electromagnetic motor of a seismic source", Journal of Mining Science, vol. 46, pp. 656-665, 2010.

[8] L.A. Neyman, V.Y. Neyman, "Dynamic model of a vibratory electromechanical system with spring linkage", 11 International forum on strategic technology (IFOST 2016): proc., Novosibirsk, 1-3 June 2016, Pt. 2, pp. 23-27, 2016.

[9] B.F. Simonov, V.Y. Neyman, A.S. Shabanov, "New conception of an electromagnetic drive for a vibration source in hole", The 18 international conference of young specialists on micro/nanotechnologies and electron devices, EDM 2017: proc., Altai, Erlagol, 29 June - 3 July 2017, pp. 507-510, 2017.

[10] E.V. Sagaradze, G.P. Svintsov, V.N. Shoffa, "Air-gap permeance of symmetric u-shaped electromagnet with prismatic cores and external armature", Russian Electrical Engineering, vol. 73, Num. 7, pp. 32-39, 2002.

[11] Y.M. Zaitsev, O.A. Petrov, N.V. Russova, G.P. Ivanov I.P. Svintsov, A.V. Prikazshchikov, "Minimizing the power consumption of a clappertype dc electromagnet in intermittent operation", Russian Electrical Engineering, vol. 86, Num. 8, pp. 474-478, 2015.

[12] Y.I. Klimenko, D.V. Batishchev, A.V. Pavlenko, V.P. Grinchenkov, "Design of a linear electromechanical actuator with an active vehicle suspension system" Russian Electrical Engineering, vol. 86, Num. 10, pp. 588-593, 2015.

[13] R.R. Sattarov, "Electromechanical transients in passive suspension systems with eddy current dampers" 9th International Conference on Power Drives Systems, ICPDS 2016, Conference Proceedings 9, p. 7756676, 2016.

[14] L.A. Neyman, V.Y. Neyman, K.A. Obukhov, "New method of the synchronous vibratory electromagnetic machine mechatronic module control", The 18 international conference of young specialists on micro/nanotechnologies and electron devices, EDM 2017: proc., Altai, Erlagol, 29 June - 3 July 2017, pp. 516-519, 2017.

[15] L.A. Neyman, V.Y. Neyman, A.S. Shabanov, "Vibration dynamics of an electromagnetic drive with a half-period rectifier", The 18 international conference of young specialists on micro/nanotechnologies and electron devices, EDM 2017: proc., Altai, Erlagol, 29 June - 3 July 2017, pp. 503-506, 2017.

[16] A.V. Prikazchikov, N.V. Russova, E.V. Sagaradze, G.P. Svintsov, D.G. Shoglev, "Improved method of design simulation of force-controlled valve electromagnet in scheme with ballast resistor", Russian Electrical Engineering, vol. 82, Num. 1, pp. 55-60, 2011. 